\title{
Pairing CT and \\ Laboratory Data to \\ Predict Prognosis \\ in COVID-19
}

\section{Katherine Colvin}

Editorial Coordinator

Citation: EMJ Radiol. 2021;2[1]:22-23.

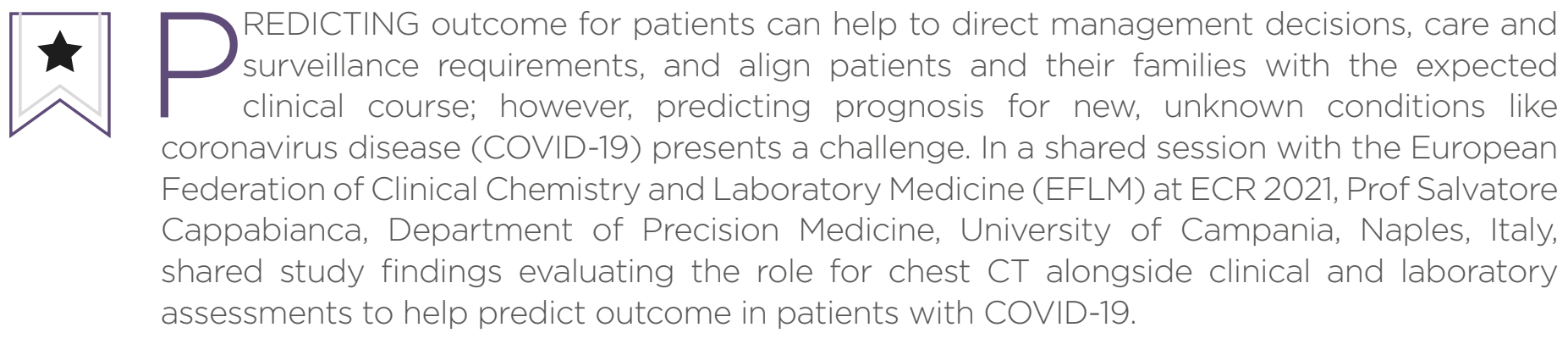

In the early period of the pandemic, Prof Cappabianca explained, studies examined the use of CT imaging for diagnosis of COVID-19; however, evolution of clinical thinking moved to support consideration of COVID-19 as a general viral infection, where the role of CT imaging is in defining extent of disease rather than diagnosis. $\mathrm{He}$ discussed his retrospective, univariate, and multivariate analysis, which aimed "to clarify the place of the CT scan in the management of COVID-19 patients, define the possibilities of CT alone in prediction of patients' outcome, and to compare lung $\mathrm{CT}$ impairment with clinical data to improve performance in outcome prediction."

The study analysed data from 103 patients presenting to hospital: 41 female and 62 male, aged 29-93 years. Patients had confirmed severe acute respiratory syndrome coronavirus 2 (SARS-CoV-2) infection via reverse transcription
PCR nasopharyngeal swab, with admission chest CT usually performed after confirmed COVID-19 diagnosis. The chest CT protocol was performed with supine positioning, without intravenous contrast, and imaged lung apices to bases using a standard dose scanning protocol $(1.25 \mathrm{~mm}$ thickness, $1 \mathrm{~mm}$ interval reconstruction). Two experienced radiologists, blinded to clinical and laboratory findings, defined the presence and extension of ground-glass opacities and areas of consolidation using a guideline for structured reporting, to provide a visual score as a severity index. This visual score reporting was supplemented with an artificial intelligence tool, which reported volumes of residual healthy lung parenchyma, ground-glass opacities, consolidation, and emphysema.

Both univariate and multivariate analyses were performed, incorporating data from 


\section{The best prediction of prognosis, however, came with combining clinical, laboratory, and CT findings.}

clinical and laboratory assessment (including oxygen saturation, procalcitonin, D-dimer, and troponin), clinical picture (such as fever, cough, and presence of respiratory failure), and comorbidities (hypertension, diabetes, cardiac and/or lung pathologies, neurological pathologies, and neoplasm). Outcomes were assessed as discharge home, hospitalisation in stable condition, and hospitalisation in critical condition.

Analysis found that age, oxygen saturations, C-reactive protein, leukocyte and neutrophil count, lactate dehydrogenase, D-dimer, troponin, creatinine and azotemia, alanine aminotransferase, aspartate aminotransferase, and bilirubin were the major clinical and laboratory findings that defined the outcome of the patient; comorbidities and symptomology showed no significant relationship with the evolution of the COVID-19 pathology.

As determined by the artificial intelligence tool, lung volumes of emphysema, residual healthy lung parenchyma, and consolidation were shown to be "highly important predictive tools in the evaluation of the evolution of disease," outlined Prof Cappabianca; volume of ground glass opacities was not a statistically significant predictor. The visual severity score also demonstrated good correlation with patient outcome.

The best prediction of prognosis, however, came with combining clinical, laboratory, and CT findings. The study found that this combined approach yielded a sensitivity, specificity, and accuracy for predicting outcome of $88 \%, 78 \%$, and $81 \%$, respectively.

Prof Cappabianca summarised the conclusions of his study for the use of chest CT in prognostication for COVID-19: "our experience suggests the usefulness of visual quantification of involved lung as a predictor of the outcome in patients affected by coronavirus disease. Comparison with clinical and laboratory data provides higher correlation with prediction and software-based quantification of consolidation, emphysema, and residual normal lung on chest CT are independent predictors of COVID-19 evolution."

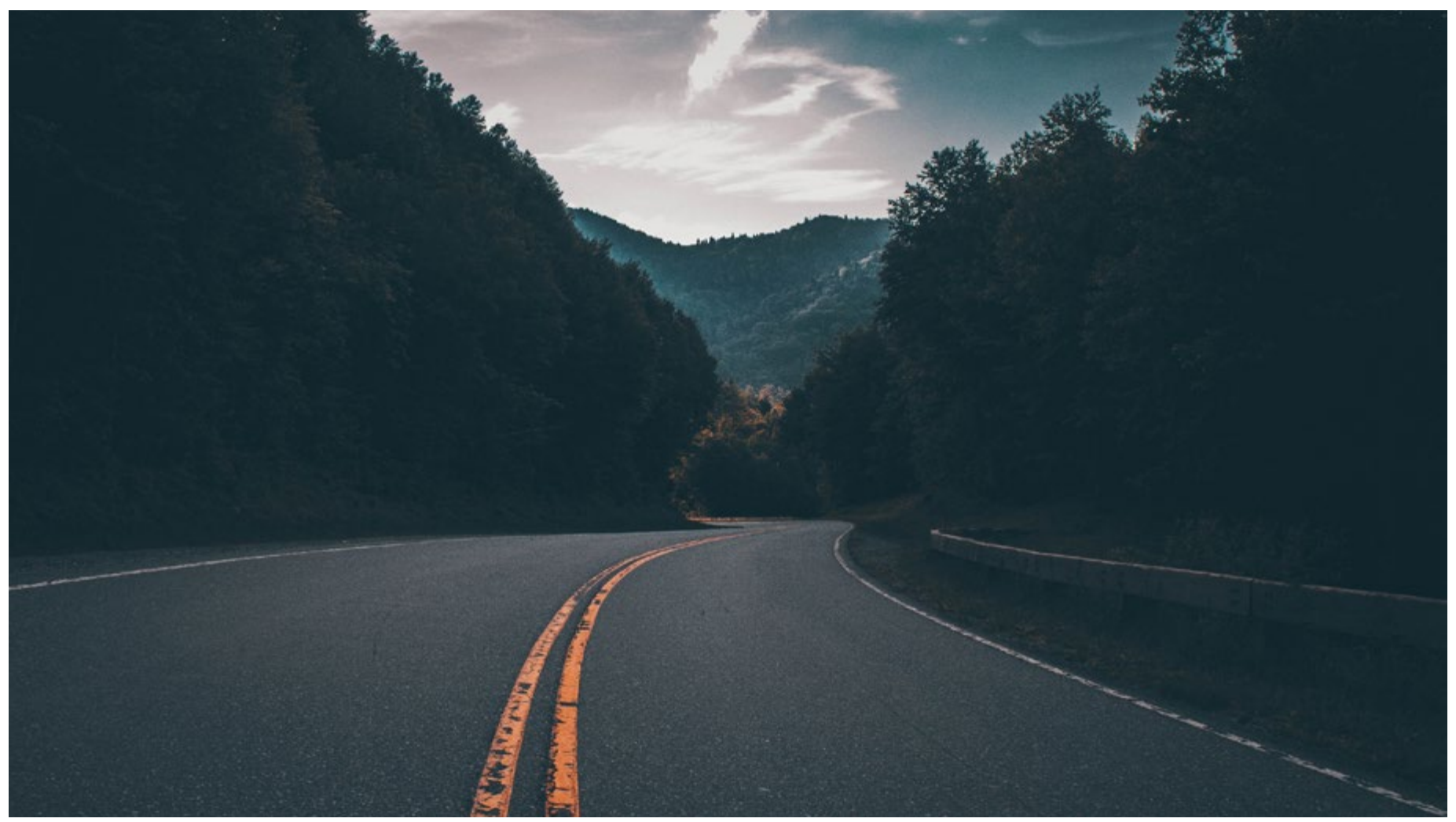

